\title{
Index Theorem for the $q$-Deformed Fuzzy Sphere
}

\author{
E. Harikumar ${ }^{a *}$ Amilcar R. Queiroz ${ }^{b \dagger}$ and P. Teotonio-Sobrinho ${ }^{b \ddagger}$ \\ ${ }^{a}$ School of Physics, University of Hyderabad, \\ Central University P O, Hyderabad-46, AP, India, Pin 500046 \\ ${ }^{b}$ Instituto de Física, Universidade de São Paulo, \\ Caixa Postal 66318, 05315-970, São Paulo, SP, Brazil
}

January 1, 2018

\begin{abstract}
We calculate the index of the Dirac operator defined on the q-deformed fuzzy sphere. The index of the Dirac operator is related to its net chiral zero modes and thus to the trace of the chirality operator. We show that for the q-deformed fuzzy sphere, a $U_{q}(s u(2))$ invariant trace of the chirality operator gives the q-dimension of the eigenspace of the zero modes of the Dirac operator. We also show that this q-dimension is related to the topological index of the spinorial field as well as to the fuzzy cut-off parameter. We then introduce a q-deformed chirality operator and show that its $U_{q}(s u(2))$ invariant trace gives the topological invariant index of the Dirac operator. We also explain the construction and important role of the trace operation which is invariant under the $U_{q}(s u(2))$, which is the symmetry algebra of the q-deformed fuzzy sphere. We briefly discuss chiral symmetry of the spinorial action on q-deformed fuzzy sphere and the possible role of this deformed chiral operator in its evaluation using path integral methods.
\end{abstract}

*harisp@uohyd.ernet.in

†amilcar@fma.if.usp.br

†teotonio@fma.if.usp.br 


\section{Introduction}

The index of Dirac operator is known to be related to topological invariance of the underlying space where it is defined. The index of a self-adjoint operator $\mathcal{A}$ is defined as the difference between the number of the zero modes of $\mathcal{A}$ and its adjoint $\mathcal{A}^{\dagger}[1$. Thus, in the case of Dirac operator, the index is a measure of the net chiral zero modes and thus it is related to the chirality operator $\Gamma$ and also to the chiral symmetry of the fermionic field theories. The study of index theory of the Dirac operator have been of interest for the understanding of chiral anomaly apart from its importance in understanding the topological features of the underlying space. For fermions coupled to gauge fields, it is known that the index can be expressed in terms of the field strength of gauge fields alone. The index is exactly the Pontryagin index which is a topological invariant quantity of the underlying space [1]. In the Fujikawa mechanism [2], this topological term is added to the classical action so as to make the the partition function invariant under the chiral transformations which is a symmetry of the classical theory [1, 2, 3].

The study of the index theorem for Dirac operators on different spaces are of interest not only for the investigation of the topological features but also for the understanding of chiral symmetry. This relation between the zero modes, topological charge of the underlying space and chiral anomaly have been analysed for Dirac operators on various spaces such as two sphere and fuzzy sphere [4, 5, 6, 7, 8].

In this paper we obtain the index theorem for a recently constructed 9] Dirac operator on the q-deformed fuzzy sphere. An important feature of this $D_{q}$ is its invariance under the action of $U_{q}(s u(2))$. Such symmetry plays a determinant role in the computation of the spectrum of $D_{q}$. The eigenfunctions of $D_{q}$ obtained in [9] can be classified into \pm chiral subspaces using the chirality operator $\Gamma$. We show a possible modification of the chirality operator obtained in [9] and this deformed chiral operator $\tilde{\Gamma}$ also splits the spinor space into \pm chiral subspace and reduces to chiral operator on fuzzy sphere in the limit $q \rightarrow 1$. Using relation of the index of $D_{q}$ to the trace of the chirality operator, we explicitly find the index of $D_{q}$. In obtaining this, we show the important role played by the $U_{q}(s u(2))$ invariant trace. We show that the trace of the chirality operator gives a relation between $q$-dimension of the eigenspace of the zero modes of $D_{q}$ and the topological index of the spinor field. We then calculate the index using the deformed chirality operator $\tilde{\Gamma}$. We observe that the indices obtained from chirality operator $\Gamma$ as well as deformed chirality operator $\tilde{\Gamma}$, have the proper limit with $q \rightarrow 1$.

The relation between Hopf algebra (also known as quantum groups) and noncommutative spaces is a well estabilished matter. In particular, the geometrical and topological aspects of these spaces have been studied in [10, 11]. The construction of field theories on noncommutative geometries with underlying symmetry being $U_{q}(s u(2))$ or its quantum dual $S U(2)_{q}$ has been explored in 12 .

The study of field theory models on non-commutative space in general and on fuzzy $S^{2}$ and $S_{q F}^{2}$ in particular, are of importance as they provide alternate, finite dimensional, regularised models. These models may have advantages such as the absence of fermion doubling problem which plague the usual lattice formulation [13. Attempts to construct gauge field theories on such manifolds have also been undertaken [14]. We hope that the understanding of the index theory and chiral invariance in these models ( à la Fujikawa mechanism ) can be helpful in providing us valuable hints in the construction of gauge theories on $S_{q F}^{2}$.

The $q$-deformed fuzzy sphere $S_{q F}^{2}(N)$ is described by a finite dimensional matrix algebra and carries an action of $U_{q}(s u(2))$. The number $N$ which fixes the dimension of the matrices is called the fuzzy cut-off parameter. In the limit $q \rightarrow 1$, this reduces to the usual fuzzy sphere defined as 
the matrix algebra invariant under $s u(2)$.

In other words, $U_{q}(s u(2))$ is the symmetry of the q-deformed fuzzy sphere just as $s u(2)$ is the symmetry of the usual fuzzy sphere. We can describe $U_{q}(s u(2))$ as the algebra generated by the operators $J_{ \pm}$and $K \equiv q^{J_{3}}$, where $q$ can be a positive real number or a root of unity (i.e., $q=e^{\frac{2 \pi i}{p}}$ with $p$ being a positive number) such that the operators satisfy the relations

$$
\left[J_{+}, J_{-}\right]=\frac{K-K^{-1}}{q^{\frac{1}{2}}-q^{-\frac{1}{2}}} \quad \text { and } \quad K J_{ \pm} K^{-1}=q^{ \pm 1} J_{ \pm} .
$$

Here we used the definition of $q$-number of $x$

$$
[x] \equiv[x]_{q}=\frac{q^{\frac{x}{2}}-q^{-\frac{x}{2}}}{q^{\frac{1}{2}}-q^{-\frac{1}{2}}} .
$$

$U_{q}(s u(2))$ being a Hopf algebra [16], the Hopf algebra structures, namely, co-product $\Delta$, antipode $S$ and co-unit $\epsilon$ of $U_{q}(s u(2))$ are given by

$$
\begin{gathered}
\Delta\left(J_{ \pm}\right)=J_{ \pm} \otimes K^{\frac{1}{2}}+K^{-\frac{1}{2}} \otimes J_{ \pm}, \quad \Delta(K)=K \otimes K \\
S\left(J_{ \pm}\right)=-K^{\frac{1}{2}} J_{ \pm} K^{-\frac{1}{2}}, \quad S(K)=K^{-1} \\
\epsilon\left(J_{ \pm}\right)=0, \quad \epsilon(K)=\epsilon(\mathbb{I})=1
\end{gathered}
$$

where $[n]_{q} !=[n]_{q}[n-1]_{q} \ldots[1]_{q}$. Observe that the Hopf algebra structures are not preserved by $q \leftrightarrow q^{-1}$. We, thus, have two Hopf algebras $U_{q}(s u(2))$ and $U_{q^{-1}}(s u(2))$.

After briefly stating the well known index theorem in a language suitable for our purpose in the next section, we summarise the essential details of the constructions of spinor module and its chiral decomposition, Dirac operator and its spectrum. This is done first for the fuzzy sphere in section 3 , where we also show the validity of index theorem and then in section 4 for the q-deformed fuzzy sphere with more details about the $U_{q}(s u(2))$-invariant trace, which will be crucial for our later discussions. In section 5 we present our main result, namely the derivation of the index theorem for the Dirac operator $D_{q}$ on $S_{q F}^{2}$. Here we show that the trace of the chirality operator $\Gamma$ relates the q-dimension of the eigenspace of $D_{q}$ to the topological index of the spinor field and also to the fuzzy cut-off. We then define a $q$-deformed chirality operator $\tilde{\Gamma}$ and show that its trace gives the topological invariant index of $D_{q}$. We conclude in section 6 with comments about the important role of $U_{q}(s u(2))$-invariant trace and its relevance to the chiral anomaly of the theory defined on $S_{q F}^{2}$.

\section{Index Theorem}

The index of an operator $D$ is defined as the difference between the number of the zero modes of $\mathrm{D}$ and that of $D^{\dagger}$, i.e.,

$$
\text { Index } D=\operatorname{dim}(\operatorname{Ker} D)-\operatorname{dim}\left(\operatorname{Ker} D^{\dagger}\right)
$$

where the $\operatorname{dim}(\mathrm{Ker})$ is the dimension of the space of zero modes of the respective operators. The index theorem relates the topological invariance of the manifold where the operator is defined to its index[1. In the case of the Dirac operator in Euclidean space, the index $\nu$ is the difference 
between $n_{+}$, the number of zero modes with positive chirality and $n_{-}$, the number of zero modes with negative chirality. It is a well known fact that the index of the Dirac operator is a topological invariant, i.e.,

$$
n_{+}-n_{-}=\nu=\frac{1}{32 \pi^{2}} \int d^{4} x \epsilon_{\mu \nu l \sigma} F^{\mu \nu} F^{l \sigma} .
$$

In order to evaluate the index of the Dirac operator, it is sufficient to find all the zero modes and classify them according to chirality. Thus we see that the index of the Dirac operator is the same as the trace of the chirality operator $\Gamma$ restricted to the space of zero modes of the Dirac operator. Here, to clarify the evaluation of the index, we start with a generic self-adjoint operator

1. $D$ which act on a vector space $V$,

2. An involution operator $\Gamma$ on $V\left(\right.$ i.e., $\Gamma^{2}=1$ ) which anti-commutes with $D$, i.e., $D \Gamma+\Gamma D=0$.

Once these conditions are fulfilled, one can see that

$$
\operatorname{Tr} \Gamma=\left|n_{+}-n_{-}\right|
$$

where the trace is evaluated on $V$ and $n_{ \pm}$are the number of \pm -chiral zero modes. We can simplify the calculation of the above trace by splitting $V$ into two subspaces with respect to the eigenvalues of $D$. Thus let $V_{0}$ be the subspace of $V$ such that $D\left(V_{0}\right)=0$ and $V_{1}$ the subspace of $V$ such that $D\left(V_{1}\right) \neq 0$, then $V=V_{0} \oplus V_{1}$. The trace can be written as a sum of two traces, one of them taken on $V_{0}$ and the other on $V_{1}$, i.e.,

$$
\operatorname{Tr}(\Gamma)=\operatorname{Tr}_{D=0}(\Gamma)+\operatorname{Tr}_{D \neq 0}(\Gamma)
$$

One can easily see that the second term on the right hand side vanishes. Indeed, if we consider the operator $|D|=\left(D D^{\dagger}\right)^{\frac{1}{2}}>0$, then

$$
\begin{aligned}
\operatorname{Tr}_{D \neq 0}(\Gamma) & =\operatorname{Tr}_{D \neq 0}\left(\frac{D}{|D|} \Gamma \frac{D^{\dagger}}{|D|}\right) \\
& =-\operatorname{Tr}_{D \neq 0}\left(\frac{D}{|D|} \frac{D^{\dagger}}{|D|} \Gamma\right)=-\operatorname{Tr}_{D \neq 0}(\Gamma),
\end{aligned}
$$

where we have used the facts $D^{\dagger}=D,|D| \Gamma=\Gamma|D|$ and $\Gamma D+D \Gamma=0$. Thus we get

$$
\operatorname{Tr}(\Gamma)=\operatorname{Tr}_{D=0}(\Gamma)=\operatorname{Tr}_{D=0}\left(\frac{1+\Gamma}{2}-\frac{1-\Gamma}{2}\right)=n_{+}-n_{-},
$$

where $\operatorname{Tr}_{D=0}$ is the usual trace on $V$ restricted to the subspace $V_{0}$ of the zero modes of the operator $D$. The number $n_{+}$is the dimension of the subspace of $V_{0}$ with positive chirality $\left(\Gamma V_{0}^{+}=V_{0}^{+}\right)$, and likewise $n_{-}$is the dimension of the subspace of $V_{0}$ with negative chirality $\left(\Gamma V_{0}^{-}=-V_{0}^{-}\right)$. Note that the operators $(1 \pm \Gamma) / 2$ project the spinors to \pm chiral subspaces and in particular on $V_{0}^{ \pm}$ respectively, in this case. 


\section{Dirac Operator on the Fuzzy Sphere}

In this section, we briefly recall the essential features of this Dirac operator and its spectrum on fuzzy sphere [15] and show the validity of the index theorem.

The Dirac operator on the fuzzy sphere maps the $s u(2)$ spinor module $\left(\mathcal{S}_{k}^{J}\right)$ to itself. The spinorial field belonging to this spinor module $\mathcal{S}_{k}^{J}$ is defined as

$$
\Psi=\Psi^{+}\left(a_{i}^{\dagger}, a_{i}, b^{\dagger}, b\right)+\Psi^{-}\left(a_{i}^{\dagger}, a_{i}, b^{\dagger}, b\right)=f\left(a_{i}^{\dagger}, a_{i}\right) b+g\left(a_{i}^{\dagger}, a_{i}\right) b^{\dagger},
$$

where $a_{i}^{\dagger}, a_{i}, i=1,2$ are two sets of bosonic creation and annihilation operators, $b^{\dagger}, b$ is a set of fermionic creation and annihilation operators $\left(\left\{b, b^{\dagger}\right\}=1\right)$, and the functions $\Psi^{ \pm}$can be written as a linear combination of monomials with fixed topological index $2 k \in \mathbb{Z}$. Thus we have

$$
\Psi^{ \pm}=\sum_{m_{1}, m_{2}, \mu, n_{1}, n_{2}, \nu} c_{m_{1}, m_{2}, \mu, n_{1}, n_{2}, \nu} a_{1}^{\dagger} m_{1} a_{2}^{\dagger m_{2}} b^{\dagger \mu} a_{1}^{n_{1}} a_{2}^{n_{2}} b^{\nu}
$$

where $m_{1}, m_{2}, n_{1}, n_{2}$ are non-negative integers and $\mu, \nu=0,1, \mu+\nu=1$ satisfying $m_{1}+m_{2}+\mu \leq M$, $n_{1}+n_{2}+\nu \leq N, M-N=2 k$ apart from the condition $m_{1}+m_{2}+\mu-n_{1}-n_{2}-\nu=2 k$. We also have $M+N=2 J$ which is the fuzzy cut-off parameter. Some properties we have to bear in mind concerning these spinorials $\Psi^{ \pm}$are

- they map the Fock space $\mathcal{F}_{N}^{\mu}$ to the Fock Space $\mathcal{F}_{M}^{\nu}$. These Fock spaces are Hilbert spaces of representations of $s u(2)$ and are given by

$$
\left|n_{1}, n_{2} ; \nu\right\rangle=\frac{1}{\sqrt{n_{1} ! n_{2} !}} a_{1}^{\dagger n_{1}} a_{2}^{\dagger n_{2}} b^{\dagger \nu}|0\rangle, \quad n_{1}+n_{2}+\nu=N
$$

- The bosonic parts of the spinor $\Psi$, i.e., $f$ and $g$ in (11), are tensor representations with even dimensions of $U(s u(2))$. Therefore, the angular momentum number $l$ of these operators are half-integers $\frac{2 s+1}{2}$, where $s \in \mathbb{Z}$. Further, the bosonic operator $f$ can be written as a $\frac{M}{2} \otimes \frac{(N-1)}{2}$ matrix, and the bosonic operator $g$ can be written as a $\frac{(M-1)}{2} \otimes \frac{N}{2}$ acting on the column vector representation of the Fock space (13). Thus $f$ and $g$ can be written in terms of the tensor operators belonging to the half-integer representations

$$
\begin{aligned}
\frac{M}{2} \otimes \frac{N-1}{2} & =\left|k+\frac{1}{2}\right| \oplus \ldots \ldots \oplus\left(J-\frac{1}{2}\right) \\
\text { and } \quad \frac{M-1}{2} \otimes \frac{N}{2} & =\left|k-\frac{1}{2}\right| \oplus \ldots \ldots \oplus\left(J-\frac{1}{2}\right)
\end{aligned}
$$

respectively where $J=\frac{M+N}{2}$.

- On the spinorial module $\mathcal{S}_{k}^{J}$ we define the chirality operator as

$$
\Gamma \Psi=-\left[b^{\dagger} b, \Psi\right]
$$

which has \pm 1 eigenvalues. We denote the respective eigenspaces as $\mathcal{S}_{k}^{J \pm}$. 
- The spinorial module is formed by the linear combination of vectors belonging to the halfinteger spin spaces, $\Phi_{J, k+\frac{1}{2}, m}^{j}$ which can be obtained by the repeated action of $J_{+}$(using the co-product) on

$$
\Phi_{J, k+\frac{1}{2},-j}^{j}=\mathcal{N} a_{2}^{\dagger\left(j+k+\frac{1}{2}\right)} a_{1}^{\left(j-k-\frac{1}{2}\right)} .
$$

This spinor module can be splitted into positive chiral and negative chiral subspaces using the above $\Gamma$. Thus we have

$$
\mathcal{S}_{k}^{J}=\mathcal{S}_{k}^{J+} \oplus \mathcal{S}_{k}^{J-}
$$

Now the Dirac operator which maps the spinor module to itself and also anti-commutes with $\Gamma$ is defined using two operators $K_{ \pm}$and acts on $\Psi$ as

$$
D \Psi=K_{+} \Psi+K_{-} \Psi .
$$

Here the $K_{ \pm}$are operators mapping $\mathcal{S}_{k}^{J \pm}$ to $\mathcal{S}_{k}^{J}{ }^{\mp}$. In terms of the operators $a_{i}^{\dagger}, a_{i}, b^{\dagger}, b$ we write the action of $K_{ \pm}$as

$$
\begin{aligned}
& K_{+} \Psi=b a_{2}^{\dagger} \Psi a_{1}^{\dagger} b-b a_{1}^{\dagger} \Psi a_{2}^{\dagger} b \\
& K_{-} \Psi=b^{\dagger} a_{1} \Psi a_{2} b^{\dagger}-b^{\dagger} a_{2} \Psi a_{1} b^{\dagger} .
\end{aligned}
$$

We can easily check that with the above definitions, the eigenfunctions of the Dirac operator are $\Psi_{J, k, m}^{j \pm}=\frac{1}{\sqrt{2}}\left[\Phi_{J, k+\frac{1}{2}, m} b \pm \Phi_{J, k+\frac{1}{2}, m} b^{\dagger}\right]$ with eigenvalues $\sqrt{\left(j+\frac{1}{2}+k\right)\left(j+\frac{1}{2}-k\right)}$. The $|M-N|$ zero modes are

$$
\begin{gathered}
\Psi_{+0}^{m_{1} m_{2}}=\mathcal{N}_{1} a_{1}^{\dagger m_{1}} a_{2}^{\dagger m_{2}} b^{\dagger} \\
\Psi_{-0}^{n_{1} n_{2}}=\mathcal{N}_{2} a_{1}^{n_{1}} a_{2}^{n_{2}} b
\end{gathered}
$$

where $\mathcal{N}_{\alpha}, \alpha=1,2$ are proportionality constants and allowed value of topological index $2 k$ for the above zero modes are $m_{1}+m_{2}+\mu>0$ and $n_{1}+n_{2}+\nu<0$ respectively and for both these zero modes, the spin $j=|k|-\frac{1}{2}$. Thus it is clear that the number of zero modes are $2 j+1=2|k|$ which is equal to $|M-N|[15]$. It is clear that the trace of $\Gamma$ restricted to the space of these zero modes of the Dirac operator will count the net chiral zero modes since each of the \pm chiral zero modes will have \pm 1 as eigenvalues under the chiral operator. We have seen that this number is equal to $2 k \in \mathbb{Z}$ which is the topological index of the spinor field by construction. Thus we see here that the index theorem is satisfied by the Dirac operator defined above on fuzzy sphere.

$D$ and $\Gamma$ as operators on the $U(s u(2))$-module $\mathcal{S}_{k}^{J}$ fulfill the conditions for the index theorem stated above and they are invariant with respect to $s u(2)$. The $s u(2)$ invariant trace is given by the usual trace on the module $\mathcal{S}_{k}^{J}$ and it is this trace we use in evaluating the index of the Dirac operator, $D$.

\section{$4 \quad U_{q}(s u(2))$ Invariant Dirac Operator $D_{q}$}

In this section we present an $U_{q}(s u(2))$ invariant Dirac operator $D_{q}$ defined on q-deformed fuzzy sphere. The $D_{q}$ and its spectrum were obtained in [9]. One of the interesting aspects of the spectrum of this Dirac operator is the novel double degeneracy for the case where $q$ is root of unity. Also in this case, we showed that there is a natural cut-off introduced by the root of unity. We now briefly recall the main points of the derivation of $D_{q}$ which are necessary for the derivation of the index theorem. 


\section{Tensor Representations of $U_{q}(s u(2))$}

A tensor representation of a Hopf algebra is a representation of this algebra on a tensor product of two vector spaces through the use of the co-product. Therefore, in the present case, if $\mathcal{H}_{l}$ and $\mathcal{H}_{l}^{*}$ are an $U_{q}(s u(2))$ irreducible representation and its dual, then the tensor representation on the space $\mathcal{H}_{l} \otimes \mathcal{H}_{l}^{*}$ is given by

$$
\rho_{\otimes}(a) \equiv \rho \otimes \bar{\rho}(\Delta(a)) \mathcal{H}_{l} \otimes \mathcal{H}_{l}^{*} \subset \mathcal{H}_{l} \otimes \mathcal{H}_{l}^{*}
$$

for $a \in U_{q}(s u(2))$ and $\rho: U_{q}(s u(2)) \rightarrow \operatorname{Aut}\left(\mathcal{H}_{l}\right)$ is a homomorphism of the algebra $U_{q}(s u(2))$ to the algebra of automorphisms of $\mathcal{H}_{l}$ and $\bar{\rho}: U_{q}(s u(2)) \rightarrow A u t\left(\mathcal{H}_{l}^{*}\right)$ is also an homorphism of algebra. If $\mathcal{H}_{l}$ is the irreducible representation of dimension $2 l+1$, then we see immediately that $\mathcal{H}_{l} \otimes \mathcal{H}_{l}^{*}$ is not irreducible. However, if $q$ is real and positive it can be always decomposed into a direct sum of irreducible representations, i.e.,

$$
\mathcal{H}_{l} \otimes \mathcal{H}_{l}^{*}=\bigoplus_{j=0}^{2 l} \mathcal{H}_{j}
$$

where $\mathcal{H}_{j}$ are the irreducible representations of dimension $2 j+1$. Note that with additional conditions, such a decomposition is possible for $q$ being root of unity also.

So, if $q=e^{\frac{2 \pi i}{p}}$, we restrict ourselves to irreducible nilpotent (or classical) representations ${ }^{1}$, in the sense that the eigenvalues of $\left(J_{+}\right)^{p},\left(J_{-}\right)^{p}$ are both zero, then the decomposition into irreducibles [18 is

$$
\mathcal{H}_{l} \otimes \mathcal{H}_{l}^{*}=\bigoplus_{j=0}^{[2 l, p-1]} \mathcal{H}_{j},
$$

where $[2 l, p-1]$ is $2 l$ if $2 l \leq p-1$ and $\frac{p-1}{2}$ otherwise.

The tensor representation has for basis the set $\left\{T_{j k}\right\}_{j=0, k=-j}^{2 l, j}$. We also denote the angular momentum quantum number $j$ as the rank of the tensor. The action of the algebra on these tensors and their eigenvalues are given by

$$
\begin{aligned}
\rho_{\otimes}\left(J_{ \pm}\right) T_{j k}=J_{ \pm} T_{j k} K^{-\frac{1}{2}}-K^{-\frac{1}{2}} T_{j k} K^{\frac{1}{2}} J_{ \pm} K^{-\frac{1}{2}} & =\sqrt{[j \pm k+1][j \mp 1]} T_{j k \pm 1}, \\
\rho_{\otimes}(K) T_{j k}=K T_{j k} K^{-1} & =q^{k} T_{j k} .
\end{aligned}
$$

Observe that these tensors act as operators on $\mathcal{H}_{l}$.

We also define the dual tensor representation. These are representations $\bar{\rho}_{\otimes} \equiv \bar{\rho} \otimes \overline{\bar{\rho}}$. We denote the irreducible basis of this dual representation by $T_{j k}^{\ddagger}$. The relation between $T_{j k}^{\ddagger}$ and $T_{j k}$ is given by

$$
T_{j k}^{\ddagger}=(-1)^{-k} q^{\frac{k}{2}} T_{j-k} .
$$

It is important to note that using these tensor operators with rank $j=\frac{1}{2}$, we can construct the tensors of higher ranks using the $q$-Clebsch-Gordan coefficients [16] ( Therefore it is enough to show the results explicitly for the $q$-tensor operators with $j=\frac{1}{2}$ ).

\footnotetext{
${ }^{1}$ If $q$ is root of unity there are two types of representations: i) nilpotent, which can be related to $s u(2)$ irreducible representations; ii) cyclical, which is a new type of representation with no analog to $s u(2)$.
} 
Let us denote by $A_{i}^{\dagger}, A_{i}$, with $i=1,2$, two sets of $q$-bosonic creation and annihilation operators. These operators satisfy the following relations

$$
\begin{aligned}
A_{i} A_{i}^{\dagger}-q^{\frac{1}{2}} A_{i}^{\dagger} A_{i} & =q^{\frac{-N_{i}}{2}} \\
{\left[N_{i}, A_{i}^{\dagger}\right] } & =A_{i}^{\dagger} \\
{\left[N_{i}, A_{i}\right] } & =-A_{i},
\end{aligned}
$$

where $A_{i}^{\dagger} A_{i}=\left[N_{i}\right]$ and $N_{i}$ is the number operator. The $q$-bosonic operators and the number operator satisfying these relations are known as $q$-Heisenberg algebra.

We can write the tensor operators with rank $j=\frac{1}{2}[16$ in terms of this $q$-bosonic operators

$$
\begin{aligned}
\alpha_{1}^{\dagger} & \equiv T_{\frac{1}{2} \frac{1}{2}}=A_{1}^{\dagger} q^{-\frac{N_{2}}{4}} \\
\alpha_{2}^{\dagger} & \equiv T_{\frac{1}{2}-\frac{1}{2}}=q^{\frac{N_{1}}{4}} A_{2}^{\dagger}
\end{aligned}
$$

and the dual representation (27) of these tensors as

$$
\begin{aligned}
& \alpha_{1} \equiv T_{\frac{1}{2}-\frac{1}{2}}^{\ddagger}=A_{1} q^{-\frac{N_{2}+1}{4}} \\
& \alpha_{2} \equiv T_{\frac{1}{2} \frac{1}{2}}^{\ddagger}=-q^{\frac{N_{1}+1}{4}} A_{2} .
\end{aligned}
$$

The set of operators $\alpha_{i}^{\dagger}, \alpha_{i}$ satisfies

$$
\begin{aligned}
\alpha_{1}^{\dagger} \alpha_{2}^{\dagger} & =q^{-\frac{1}{2}} \alpha_{2}^{\dagger} \alpha_{1}^{\dagger} \\
\alpha_{1} \alpha_{2} & =q^{\frac{1}{2}} \alpha_{2} \alpha_{1} \\
\alpha_{1}^{\dagger} \alpha_{2} & =\alpha_{2} \alpha_{1}^{\dagger} \\
\alpha_{1} \alpha_{2}^{\dagger} & =\alpha_{2}^{\dagger} \alpha_{1} .
\end{aligned}
$$

\section{Spinor Module}

The spinor module $\mathcal{S}$ is made of half-integer angular momentum tensor operators combined with a set of fermionic creation and annihilation operators $b^{\dagger}, b$. This set of fermionic operators is the usual ones, i.e., $\left\{b, b^{\dagger}\right\}=1$. A basis of this spinorial module is a monomial of the form

$$
\alpha_{1}^{\dagger m_{1}} \alpha_{2}^{\dagger m_{2}} b^{\dagger \mu} \alpha_{1}^{n_{1}} \alpha_{1}^{n_{2}} b^{\nu} \propto A_{1}^{\dagger m_{1}} A_{2}^{\dagger m_{2}} b^{\dagger \mu} A_{1}^{n_{1}} A_{1}^{n_{2}} b^{\nu}
$$

where the proportionality constant would involve $q$ and $N_{\alpha}$. In the above $m_{1}, m_{2}, n_{1}, n_{2}$ are nonnegative integers and $\mu=\nu=0,1, \mu+\nu=1$. Also $m_{1}+m_{2}+\mu \leq M$ and $n_{1}+n_{2}+\nu \leq N$, $M-N=2 k, m_{1}+m_{2}+\mu-n_{1}-n_{2}-\nu=2 k$. We also have $M+N=2 J$ which act as the fuzzy cut-off parameter. Similar to the previous section, we define the spinorial fields as

$$
\Psi=\Psi^{+}\left(A_{i}^{\dagger}, A_{i}, b^{\dagger}, b\right)+\Psi^{-}\left(A_{i}^{\dagger}, A_{i}, b^{\dagger}, b\right)=f\left(A_{i}^{\dagger}, A_{i}\right) b+g\left(A_{i}^{\dagger}, A_{i}\right) b^{\dagger},
$$

such that

$$
\Psi^{ \pm}=\sum_{m_{1}, m_{2}, \mu, n_{1}, n_{2}, \nu} c_{m_{1}, m_{2}, \mu, n_{1}, n_{2}, \nu} A_{1}^{\dagger m_{1}} A_{2}^{\dagger m_{2}} b^{\dagger \mu} A_{1}^{n_{1}} A_{2}^{n_{2}} b^{\nu}
$$


where $c_{m_{1}, m_{2}, n_{1}, n_{2}}$ are complex numbers multiplied by factors involving $q$ and $N_{\alpha}$. The bosonic part of $\Psi$ in Eqn. (40), i.e., $f$ and $g$ can be expressed in terms of the spin-half tensors belonging to $\frac{M}{2} \otimes \frac{(N-1)}{2}$ and $\frac{(M-1)}{2} \otimes \frac{N}{2}$ respectively and can be decomposed into the direct sum of IRR of half-integer spins as shown in Eqns. (14) and (15). The spinorial field $\Psi$ are build by taking the linear combinations of the operators belonging to these spin-half spaces $\Phi_{J, k+\frac{1}{2}, m}^{j}$ where the lowest weight state is given by

$$
\Phi_{J, k+\frac{1}{2}-j}^{j}=\left(A_{2}^{\dagger} q^{\frac{N_{1}}{4}}\right)^{(j+k)}\left(A_{1} q^{-\frac{N_{2}+1}{4}}\right)^{(j-k)} .
$$

\section{$q$-Deformed Dirac Operator}

The chirality operator is defined as in the usual $s u(2)$ case

$$
\Gamma_{q} \Psi \equiv \Gamma \Psi=-\left[b^{\dagger} b, \Psi\right]
$$

where the subscript $q$ is used to distinguish it from the chirality operator on fuzzy sphere and for comparison of results in the $S_{q F}^{2}$ case to the usual fuzzy one. This chirality operator splits the $U_{q}(s u(2))$ spinor module into \pm -chiral subspaces.

As in the usual $s u(2)$ case we construct auxiliary operators $K_{ \pm}$, which map \pm chiral subspace to $\mp$ chiral subspace. We require also that these operators be invariant with respect to $U_{q}(s u(2))$. In [9], it was shown that the operators that fulfill these conditions are

$$
\begin{aligned}
& K_{+} \Psi=q^{-\frac{k-m}{4}} K^{-\frac{1}{2}} b\left[A_{1}^{\dagger} \Psi A_{2}^{\dagger} q^{\frac{k}{2}}-A_{2}^{\dagger} \Psi A_{1}^{\dagger}\right] b \\
& K_{-} \Psi=q^{-\frac{k-m}{4}} b^{\dagger}\left[A_{1} \Psi A_{2} q^{\frac{k}{2}}-A_{2} \Psi A_{1}\right] b^{\dagger} K^{-\frac{1}{2}} .
\end{aligned}
$$

The $q$-deformed fuzzy Dirac operator $D_{q}$ is defined by

$$
D_{q} \Psi=K_{+} \Psi+K_{-} \Psi
$$

and thus $D_{q}$ anti-commutes with the chirality operator $\Gamma_{q}$. It is easy to see that the zero modes of this $q$-Dirac operator are given by 9 ]

$$
\begin{aligned}
\Psi_{0+}^{m_{1} m_{2}} & =\mathcal{N}_{1} A_{1}^{\dagger m_{1}} A_{2}^{\dagger m_{2}} b^{\dagger} \\
\Psi_{0-}^{n_{1} n_{2}} & =\mathcal{N}_{2} A_{1}^{n_{1}} A_{2}^{n_{2}} b .
\end{aligned}
$$

where $\mathcal{N}_{\alpha}$, with $\alpha=1,2$ are normalisation constants and they involve factors of $q$ and the number operators $N_{\alpha}$. These zero modes correspond to the angular momentum number $j=|k|-\frac{1}{2}$. The number of these zero modes are given by $|M-N|=2 j+1=2|k|$. The non-zero eigenvalues of this Dirac operator are $\sqrt{\left[j+\frac{1}{2}+k\right]\left[j+\frac{1}{2}-k\right]}$ and corresponding eigenfunctions are

$$
\Psi_{J, k, m}^{j \pm}=\frac{1}{\sqrt{2}}\left[\Phi_{J, k+\frac{1}{2}, m} b \pm \Phi_{J, k+\frac{1}{2}, m} b^{\dagger}\right]
$$

where $\Phi_{J, k \pm \frac{1}{2}, m}$ is obtained from Eqn.(42). Here we note that the eigenvalues are real. This is true even for the case of $q=e^{\frac{2 \pi i}{p}}$ provided $2 l+1<p$ which is a constraint one naturally introduce on the fuzzy cut-off parameter [9]. This condition is also needed to ensure the positivity of the inner product as explained in the next section. The reality of the eigenvalues clearly shows that our Dirac operator is self-adjoint as required. We also note that the chirality operator $\Gamma$ anti-commutes with the Dirac operator and it splits the spinor module into \pm -chiral subspaces. 


\section{Index Theorem for $D_{q}$}

In the previous section we have presented the $U_{q}(s u(2))$ invariant Dirac operator which anticommutes with a chiral operator $\Gamma_{q} \equiv \Gamma$ and we have given its zero modes $[9]$. Both $D_{q}$ and $\Gamma_{q}$ act on an $U_{q}(s u(2))$ spinorial module $\mathcal{S}$. In order to apply the index theorem to these operators we have to first define an $U_{q}(s u(2))$ invariant trace which is also essential in showing that $D_{q}$ is self-adjoint.

$U_{q}(s u(2))$-invariant Trace

A trace acting on operators of a Hopf algebra module $V$ is said to be invariant if it satisfies

$$
\operatorname{Tr}\left(\rho_{\otimes}(\Delta(a)) \hat{T}\right)=\epsilon(a) \operatorname{Tr}(\hat{T}),
$$

where $\epsilon(a)$ is the co-unit of the element $a$ of the Hopf algebra and $\hat{T}$ is an element of vector space $V \otimes V^{*}$.

We can easily see that since the co-product of $U_{q}(s u(2))$ is deformed, the above condition for the usual trace on the $U_{q}(s u(2))$-module $\mathcal{H}_{l}$ is not satisfied. In order to have an invariant trace on a representation $\mathcal{H}_{l}$, we need to deform the trace as well. The general recipe to construct this deformed trace, which is called $q$-trace here, is given in [17. There it is shown that the usual trace is a linear functional on the space $\mathcal{H}_{l} \otimes \mathcal{H}_{l}^{*}$. However, in general, for quasi-triangular Hopf algebras this trace is not invariant. So a new trace is defined to be a linear functional on the space $\mathcal{H}_{l}^{* *} \otimes \mathcal{H}_{l}^{*}$. It can be shown that $\mathcal{H}_{l}^{* *} \simeq \mathcal{H}_{l}$ as a representation for these quasi-triangular Hopf algebras.

In the present case of $U_{q}(s u(2))$, the equivalent map $\mathcal{H}_{l} \otimes \mathcal{H}_{l}^{*}$ to $\mathcal{H}_{l}^{* *} \otimes \mathcal{H}_{l}^{*}$ is given by multiplication by the matrix representation of the generator $K=q^{J_{3}}$, i.e., the tensor basis $T_{l m} \in \mathcal{H}_{l} \otimes \mathcal{H}_{l}^{*}$ goes to $K \cdot T_{l m} \in \mathcal{H}_{l}^{* *} \otimes \mathcal{H}_{l}^{*}$. Thus, we define the $q$-trace, denoted by $\operatorname{Tr}_{q}$, as

$$
\operatorname{Tr}_{q}\left(T_{l m}\right)=\operatorname{Tr}\left(K T_{l m}\right)
$$

where in the right hand side we have the usual matrix trace[7. Now, one can easily check the invariance of this trace with respect to $U_{q}(s u(2))$. Indeed

$$
\operatorname{Tr}\left(\overline{\bar{\rho}} \otimes \bar{\rho}(\Delta(a))\left(K T_{l m}\right)\right)=\epsilon(a) \operatorname{Tr}\left(K T_{l m}\right) .
$$

Observe that we have to consider the proper representation with double bar in the first term of the tensor product. We also note that $\overline{\bar{\rho}}(a)=\rho\left(S^{2}(a)\right), S$ being the antipode.

In the space $\mathcal{H}_{l} \otimes \mathcal{H}_{l}^{*}$ we can define a positive definite inner product using the $q$-trace as

$$
(A, B)=\operatorname{Tr}_{q}\left(A^{\ddagger} B\right),
$$

where $A, B \in \mathcal{H}_{l} \otimes \mathcal{H}_{l}^{*}$ and $A^{\ddagger}$ is in the dual of $\mathcal{H}_{l} \otimes \mathcal{H}_{l}^{*}$.

It can be easily seen that the reality condition $\overline{(A, B)}=(B, A)$ is satisfied for the case of $q$ being real. But for $q$ being root of unity, it is satisfied only if we define the inner product with

$$
A^{\circledast}=K^{-1} A^{\ddagger},
$$

such that $A^{\circledast \circledast} \neq A$ and

$$
(A, B)=\operatorname{Tr}_{q}\left(A^{\circledast} B\right)=\operatorname{Tr}\left(A^{\ddagger} B\right),
$$


where on the right hand side $T r$ is the usual trace. Thus we see that the reality condition is satisfied for $q$ being root of unity also. Further we observe that the positivity of this inner product is only satisfied if $2 l+1<p$, where $q=e^{\frac{2 \pi i}{p}}$.

Thus we have now defined $U_{q}(s u(2))$-invariant trace which allows one to define the self-adjoint of a given operator and are in a position to evaluate the trace of the chirality operator on $S_{q F}^{2}$ and obtain the index theorem.

\section{Index Theorem for $D_{q}$}

The $U_{q}(s u(2))$ invariant self-adjoint Dirac operator $D_{q}$ and the chiral operator $\Gamma_{q}$ on q-deformed fuzzy sphere do satisfy all the requirements listed for a generic self-adjoint operator in section 2 . We have also presented a $q$-trace (51) and the definition of self-adjointness with respect with this new trace. Now, we are in position to apply the index theorem to the operator $D_{q}$. We first calculate the $q$-trace of $\Gamma$ and then the $q$-trace of a deformed opertator $\tilde{\Gamma}$, which we will define.

1. Invariant trace of Chiral operator $\left(\Gamma_{q}\right)$ and Index

First we consider chirality operator defined on the q-deformed fuzzy sphere given in Eqn. (43) to derive the index. Using the definition of the invariant trace and zero modes we get

$$
\operatorname{Tr}_{q, D=0}\left(\Gamma_{q}\right)=\left[n_{+}\right]-\left[n_{-}\right]
$$

This can be re-expressed using identities involving the $q$-numbers [16] as

$$
\left[n_{+}\right]-\left[n_{-}\right]=\frac{\left[n_{+}+n_{-}\right]\left[\frac{\left(n_{+}-n_{-}\right)}{2}\right]}{\left[\frac{\left(n_{+}+n_{-}\right)}{2}\right]} .
$$

Since $n_{+}-n_{-}$is the number of net chiral zero modes of $D_{q}$ which is equal to $2 k$ and $n_{+}+n_{-}=$ $2 J(2 J=M+N$ is the fuzzy cut-off paremeter $)$, we get

$$
\operatorname{Tr}_{q, D=0}\left(\Gamma_{q}\right)=\frac{[2 J][k]}{[J]} .
$$

Observe that when $q \rightarrow 1$, we get $\operatorname{Tr}(\Gamma)=2 k$ which is the correct result in this limit. Thus we observe that unlike in the case of usual fuzzy sphere, here the trace of $\Gamma_{q}$ gives $q$-number of the topological charge multiplied by factors which depend on the sum of $n_{ \pm}$. Thus we note that the trace of $\Gamma_{q}$ do not give just the count of net chiral zero modes in the case of $S_{q F}^{2}$.

The $q$-trace of the identity operator on a vector space is also known as the $q$-dimension or quantum dimension [17, 18, of this vector space. This quantity plays an important role in the representation theory of quantum groups [18. Therefore, we can see the left hand side of Eqn. (57) as the difference of the $q$-dimensions of the subspaces of positive and negative chiralities of the spinor module $\mathcal{S}$, respectively. The right hand side is a quantity depending on the topological number $k$ of the spinorial field. We can thus consider a generalization of the definition (6) as

$$
q \operatorname{Index} D \equiv q \operatorname{dim}(\operatorname{Ker} D)-q \operatorname{dim}\left(\operatorname{Ker} D^{\dagger}\right)
$$


where $q \operatorname{dim}(\mathrm{Ker})$ is the $q$-dimension of the space of zero modes of the respective operators. Then, Eqn. (57) can be written as

$$
q \operatorname{Index} D=\frac{[2 J][k]}{[J]} .
$$

Here we note that the q-index depends on the fuzzy cut-off parameter $2 J=M+N$. This novel feature of the q-deformed fuzzy sphere is absent in the usual fuzzy sphere where the fuzzy cut-off is not known to have any effect on the topological properties.

2. Invariant trace of deformed Chiral operator $(\tilde{\Gamma})$ and Index

Now we consider a deformed chirality operator $\tilde{\Gamma}=K^{-1} \Gamma$. Since $D K=K D$ we have $D K^{-1} \Gamma+K^{-1} \Gamma D=0$. Though this deformed chiral operator $K^{-1} \Gamma$ is not an involution ( $K^{-1} \Gamma \neq 1$ ), we note that it also splits the spinor module into \pm chiral subspaces and in the limit $q \rightarrow 1$, it correctly reduces to the chirality operator on $S_{F}^{2}$ as required. Due to the $K^{-1}$ factor, when acted on the chiral spinors, it picks up and extra $q^{-m}$ factor, apart from the \pm 1 . With this deformed chirality operator we find that

$$
\operatorname{Tr}_{q, D=0}\left(K^{-1} \Gamma\right)=n_{+}-n_{-}=2 k .
$$

Thus we see that the trace of the deformed chiral operator do give the net number of chiral zero modes as in the usual case. We also know that by construction 9] this difference in the number of \pm chiral zero modes is equal to the topological index $2 k$ of the spinorial field. Since here the trace have no dependency on fuzzy cut-off or $q$, we are guaranteed to get the correct result in the limit of $S_{F}^{2}$ and also in the limit of continuum sphere.

Thus we see that the trace of chirality operator $\Gamma$ as well as that of deformed chiral operator $\tilde{\Gamma}$ do reproduce the expected result in the limit of $q \rightarrow 1$. But for generic $q$, it is $\tilde{\Gamma}$ which gives the count of net chiral zero modes. Thus it seems that the deformed chiral operator may be more natural and of more use in the study of chiral anomaly on deformed fuzzy sphere.

\section{Conclusion}

In this paper we have obtained the index of the Dirac operator defined on q-deformed fuzzy sphere $S_{q F}^{2}$. Since the index is a measure of the net chiral zero modes, we calculate it by evaluating the trace of the chirality operator defined on $S_{q F}^{2}$. For this calculation we have used the $U_{q}(s u(2))$ invariant trace [7, 17. We have shown that the trace of chiral operator $\Gamma$ is proportional to the $\left[n_{+}-n_{-}\right]_{q}$. The proportionality constant depends on the q-number of the fuzzy cut-off parameter. This situation is strickingly different from the undeformed fuzzy sphere where the cut-off parameter do not affect the topological invariants of the manifold. Using the explicit form of zero modes of the Dirac operator $D_{q}$ on $S_{q F}^{2}$, we exhibit that $n_{+}-n_{-}=2 k$ where $2 k \in \mathbb{Z}$ is the topological index of the spinor field. Thus, with this chirality operator, we get the index of Dirac operator proportional to the q-number of the topological index. Since the $U_{q}(s u(2))$ invariant trace of the chirality operator is the $q$-dimension of the eigenspace of $D_{q}$, this shows that the $q$-dimension of the eigenspace is related to the topological index of the spinor field. Since the q-number reduces to usual number in the limit $q \rightarrow 1$, we see that $\left[\frac{n_{+}-n_{-}}{2}\right]_{q} \rightarrow k$ in this limit as required. Here 
we have seen that the proportionality constant depend on the sum of total chiral zero modes and in the limit this proportionality constant becomes unity. Then we have shown that a deformed chirality operator can be used in place of $\Gamma$ and its $U_{q}(s u(2))$ invariant trace is just $2 k$ as in the usual case. Though this deformed chiral operator $\tilde{\Gamma}$ is not an involutive operator, it does split the spinor module into \pm chiral subspace and also reduces to the correct chiral operator in the $q \rightarrow 1$ limit.

Using the $U_{q}(s u(2))$ invariant trace, we can construct the invariant spinorial action on qdeformed fuzzy sphere $S_{q F}^{2}$. For this, we can use $q$-Clebsch-Gordan techniques and combine $\Psi$, $D_{q} \Psi$ and their duals defined using the invariant trace. Therefore terms like $\Psi^{\ddagger} \Psi$ and $\Psi^{\ddagger} D_{q} \Psi$ are invariants with respect to $U_{q}(s u(2))$ (For showing these we use Eqn. (27)). Thus the $U_{q}(s u(2))$ invariant spinorial action is finally given by

$$
S=\frac{2 \pi R^{2}}{[N+1]} \operatorname{Tr}_{q}\left(\bar{\Psi} D_{q} \Psi+V(\bar{\Psi} \Psi)\right)
$$

where, $\bar{\Psi}=\Psi^{\ddagger}$, see (152), or $\bar{\Psi}=\Psi^{\circledast}$, see (154), depending on whether $q$ is real or root of unity, $[N+1]$ is the normalisation factor, $R$ is the radius of the underlying sphere and $V(\bar{\Psi} \Psi)$ is a potential function on the $U_{q}(s u(2))$ invariant $\bar{\Psi} \Psi$. It can be easily seen that the above action is also invariant under chiral transformations for the massless case. Now, one may ask if the chiral symmetry of the action is still a symmetry of the corresponding quantum theory.

Consider the chiral invariant action $\mathcal{S}=\left.S\right|_{V=0}$ where the action $S$ is given in Eqn. (61). The corresponding partition function is

$$
\mathcal{Z}=\int \mathcal{D} \bar{\Psi} \mathcal{D} \Psi e^{-\mathcal{S}}
$$

It is not invariant with respect to the chiral transformations ${ }^{2}$

$$
\Gamma: \Psi \rightarrow e^{i \alpha \Gamma} \Psi, \quad \Gamma: \bar{\Psi} \rightarrow \Psi e^{i \alpha \Gamma}
$$

where $\alpha$ is a real parameter. As in the case of spinorial action in the commutative space we note that the contributions from \pm chiral non-zero modes to the Jacobian of chiral transformation cancel each other. But the contribution coming from the zero modes do add up to give

$$
\begin{aligned}
\mathcal{D} \bar{\Psi} \mathcal{D} \Psi & \rightarrow e^{\mid n_{+}-n_{-}} \mid \mathcal{D} \bar{\Psi} \mathcal{D} \Psi \\
& =e^{2 k} \mathcal{D} \bar{\Psi} \mathcal{D} \Psi
\end{aligned}
$$

which breaks the chiral symmetry of the quantum theory. We note here that the above contribution to the integration measure is same for the chiral transformations generated by $\Gamma$ as well as $\tilde{\Gamma}$. Thus we see that by defining an effective action with a counter-term that cancels the above contribution, one can cancel the anomaly and retain the chiral invariance. Since this new term has to be invariant under $U_{q}(s u(2))$, we need to define this term using $U_{q}(s u(2))$ invariant trace. Also since the extra phase factor is just $2 k$ and not proportional to $[k]_{q}$ we see that the effective action depend on the deformed chirality operator $\tilde{\Gamma}$ rather than the chirality operator $\Gamma_{q}$. Thus we get the effective action to be

$$
\mathcal{S}_{\text {eff }}=\mathcal{S}-\operatorname{Tr}_{q}\left(\bar{\Psi} K^{-1} \Gamma \Psi\right)
$$

\footnotetext{
${ }^{2}$ The chiral transformations effected by the deformed chiral operator $\tilde{\Gamma}=q^{-J_{3}} \Gamma$ is $\tilde{\Gamma} \cdot \Psi=\Delta\left(q^{-J_{3}}\right) e^{i \alpha \Gamma}(\Psi)$ where $\Delta\left(J_{3}\right)$ acts on the bosonic part, i.e., $f$ and $g$ of $\Psi$ and $\Gamma$ acts on the fermionic part as in Eqn. 63.
} 
Therefore, the deformed chirality operator may be better suited for the study of spinor fields and their actions on $S_{q F}^{2}$.

In the commutative spaces, the new term in the effective action in Eqn. (65) have been expressed in terms of the gauge fields alone 1 . There have been some studies aiming in the construction of gauge field theories on fuzzy sphere[14. The path integral evaluation of above term can lead to better understanding of the construction of gauge field action on q-deformed fuzzy sphere as well as on fuzzy sphere.

\section{ACKNOWLEDGMENTS}

We thank A. P. Balachandran for many useful discussions and comments. ARQ thanks FAPESP for support through the grant 02/03247-2. EH thanks V. O. Rivelles for hospitality and support at the Instituto de Física, Universidade de São Paulo, Brazil.

\section{References}

[1] W. Dittrich and M. Reuter, 'Selected Topics in Gauge Theories', Spinger-Verlag,1986, Berlin.

[2] K. Fujikawa, 'Path Integral Measure for Gauge Invariant Fermion Theories', Phys. Rev. Lett. 42, (1979) 1195; K. Fujikawa, 'Path Integral For Gauge Theories with Fermions', Phys. Rev. D 21, (1980) 2848 [Erratum- ibid. D 22, (1980) 1499].

[3] M. E. Peskin and D. V. Schroeder, 'An Introduction to Quantum Field Theory', Perseus Books, 1995, Cambridge, Massachusetts.

[4] P. Presnajder, 'The Origin of Chiral Anomaly and the Noncommutative Geometry', J. Math. Phys. 41 (2000) 2789, hep-th/9912050.

[5] A. P. Balachandran and S. Vaidya, 'Instantons and Chiral Anomaly in Fuzzy Physics', Int. J. Mod. Phys. A16 (2001) 17, hep-th/9910129 S. Baez, A. P. Balachandran, S. Vaidya and B. Ydri, 'Monopoles and Solitons in Fuzzy Physics', Commun. Math. Phys. 208 (2000) 787, hep-th/9811169 A. P. Balachandran, G. Immirzi, J. Lee, P. Presnajder, 'Dirac Operators on Coset Spaces', J. Math. Phys. 44 (2003) 4713, hep-th/0210297.

[6] U. Carow-Watamura and S. Watamura, 'Chirality and Dirac Operator on Noncommutative Sphere', Commun. Math. Phys. 183 (1997) 365, hep-th/9605003 ibid, 'Differential Calculus on Fuzzy Sphere and Scalar Field', Int. J. Mod. Phys. A 13 (1998) 3235.

[7] P. N. Bibikov, P. P. Kulish, 'Dirac operators on quantum $S U(2)$ group and quantum sphere', q-alg/9608012

[8] H. Grosse and C. W. Rupp, 'A Remark on Topological Charges over the Fuzzy Sphere', math-ph/0103003.

[9] E. Harikumar, Amilcar R. Queiroz, P. Teotonio-Sobrinho, 'Dirac operator on the q-deformed Fuzzy sphere and Its spectrum', hep-th/0603193. 
[10] L. Dabrowski, G. Landi, A. Sitarz, W. van Suijlekom and J. C. Varilly, 'The Dirac operator on $S U_{q}(2)$ ', Commun. Math. Phys. 259 (2005) 729, math.QA/0411609, W. van Suijlekom, L. Dabrowski, G. Landi, A. Sitarz and J. C. Varilly, 'Local index formula for $S U_{q}(2)$ ', K-Theory 35 (2005) 375, math.QA/0501287

[11] F. D'Andrea, L. Dabrowski, G. Landi and E. Wagner, 'Dirac operators on all Podles quantum spheres', math.QA/0606480.

[12] H. Grosse, J. Madore and H. Steinacker, 'Field Theory on the q-deformed Fuzzy Sphere I', J. Geom. Phys. 38 (2001) 308, hep-th/0005273 H. Steinacker, 'Aspects of the q-deformed Fuzzy Sphere', Mod. Phys. Lett. A16 (2001) 361, hep-th/0102074 H. Grosse, J. Madore and H. Steinacker, 'Field Theory on the q-deformed Fuzzy Sphere II: Quantization', J. Geom. Phys. 43 (2002) 205, hep-th/0103164.

[13] A. P. Balachandran, T. R. Govindarajan and B. Ydri, 'The Fermion Doubling Problem and Noncommutative Geometry', Mod. Phys. Lett. A15 (2000) 1279, hep-th/9911087; ibid, Fermion doubling problem and noncommutative geometry II, hep-th/0006216.

[14] H. Steinacker, 'Quantized Gauge Theory on the Fuzzy Sphere as Random Matrix Model', Nucl. Phys. B679 (2004) 66, hep-th/0307075.

[15] H. Grosse, C. Klimcik and P. Presnajder, 'Topologically nontrivial field configurations in noncommutative geometry', Commun. Math. Phys.180 (1996) 429, hep-th/9510083.

[16] L. C. Biedenharn and M. A. Lohe, 'Quantum Group Symmetry and q-Tensor Algebras', World Scientific, 1995; T. Curtright, D. Fairlie and C. Zachos (Eds), 'Quantum Group'( Proceedings of the Argonne Workshop), World Scientific, 1991 and references therein.

[17] V. Chari and A. Pressley, 'A Guide to Quantum Groups', Cambridge University Press, 1994.

[18] L. Alvarez-Gaume, C. Gomez and G. Sierra, 'Duality and Quantum Groups', Nucl. Phys. B330 (1990) 347. 